\title{
Performance of BEBE-prototype: A BEam-BEam counter proto- type for the MPD-NICA experiment at JINR
}

\author{
Cristian Heber Zepeda Fernández ${ }^{1, \star}$ \\ ${ }^{1}$ Centro de Investigación y de Estudios Avanzados del I.P.N.
}

\begin{abstract}
In this work we show the arrival time resolution for the Beam Monitoring Detector (BMD). We made the study for Au+Au collision at $\sqrt{s}=8 \mathrm{Gev}$ and a smearing of $\sigma=300 \mathrm{~cm}$. The arrival time resolution we found is $\Delta \sigma=57.982 \pm 0.509 \mathrm{ps}$. We show preliminary results of the time resolution for a cell of the BMD.
\end{abstract}

\section{Introduction}

The Nuclotron-based Ion Collider fAcility (NICA) is a new accelerator at the Joint Institute for Nuclear Research (JINR) in Dubna, Russia. Its field of study will be the properties of dense baryonic matter. NICA will have three experiments (or detectors):

1. The Baryonic Matter at Nuclotron (BM@N).

2. The Spin Physics Detector (SPD).

3. The MultiPurpose Detector (MPD).

Each one has its own physics of study. In Figure 1 is shown a representation of NICA.

In this report we show the work we did for MPD, which consisting of the implementation of a new detector (see sections below). The MPD (as its name says) has several physics studies. One of the principal field is to measurements of the production of strange particles (particles conformed by the strange quark). This study is due to that they can give information about the medium and particle production mechanism. To know more about the detectors of MPD see [1].

To make MPD measurements more accurate, will be added a new detector: The Beam Monitoring Detector (BMD), which will be two scintillator detectors. The main goal of BMD will be increase the pseudorapidity acceptance of MPD. Other measurements that BMD can do, are listed below.

- Optimization of events: Plane resolution.

- Centrality.

- Interaction point location.

- Multiplicity reference estimator.

- Trigger system.

\footnotetext{
^e-mail: hzepeda@fis.cinvestav.mx
} 


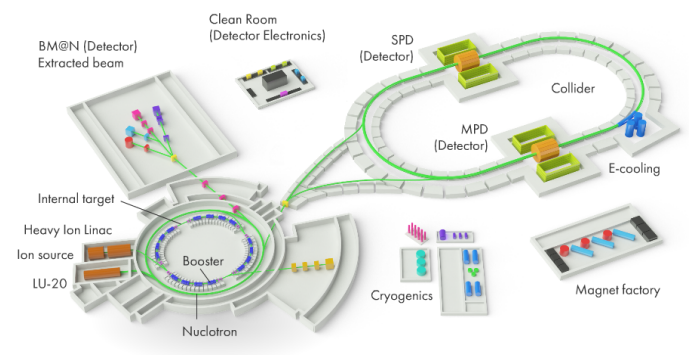

Figure 1. A picture of NICA. It is shown the three experiments and the rest of the components.

- Beam monitoring.

- Discriminate centrality events from background and beam-gas interaction.

- Determinate the absolute cross section of reaction process.

The name we will use to refer to the two detectors are "BMD-A" and "BMD-C". In the next section the properties of BMD will be descibed.

\section{Characteristics of BMD}

BMD-A and BMD-C will be located at $-200 \mathrm{~cm}$ and $200 \mathrm{~cm}$, respectively, with respect to the geometric center of MPD. For this study we used a circular geometry for each detector, whose minimum radius is $5.1 \mathrm{~cm}$, maximum radius is $73.63 \mathrm{~cm}$ and the width is $1.25 \mathrm{~cm}$, for each one. Each detector is divided in 5 rings and at the same time each ring is divided into 16 slices, giving a total of 160 scintillator pieces for all BMD. The Ring 1 is located in a radius range of $(5.1,8.30) \mathrm{cm}$, the Raidus 2 in $(8.5,14.5) \mathrm{cm}$, the Radius 3 in $(14.7,23.4) \mathrm{cm}$, the Radius 4 in $(23.6,42.00) \mathrm{cm}$ and Radius 5 in $(42.2,73.63) \mathrm{cm}$. With this geometry, the pseudorapidity range for BMD is $1.69<|\eta|<4.36$. There are 4 important studies that we have to do for BMD:

- Time resolution and arrival time resolution.

- Centrality and multiplicity determination.

- Trigger efficiencies.

- Background reaction.

The results of these studies will be able to give us a good "way" to construct the real BMD. For the present work, we made the study for the arrival time resolution.

\section{BMD simulation and results}

We simulated Au-Au collision at $\sqrt{s}=8 \mathrm{GeV}$. We do not restrict that the collision occurs at the origin, i.e., we let the collision occurs with a Gaussian distribution whose center is the geometrical origin and has a sigma of $300 \mathrm{~cm}$, this is called "smearing". We are interested in the analysis for the arrival particles to BMD-A and BMD-C. In this section we will describe the technique we used to calculate the arrival time resolution for BMD. We used the mpdroot framework for the MPD simulation.

The first result is to locate the position of interaction point. This can be possible using the addition and difference time of arrival of the particles, when the collision occurs inside or outside of BMD. The 


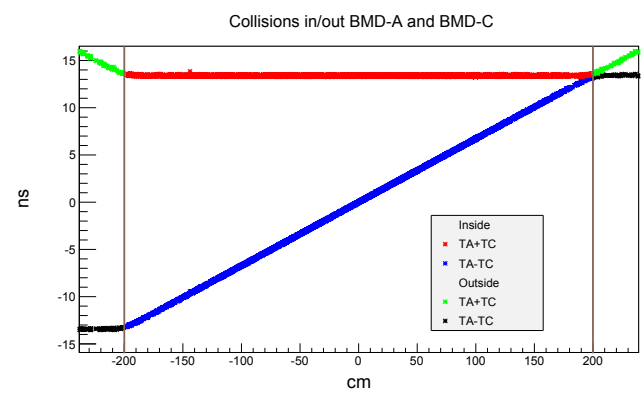

Figure 2. Comparation between TA+TC and TA-TC when the collision occurs inside and outside BMD. The vertical lines indicate the region of BMD-A and BMD-C.

position of BMD can define four zones: 1) $\left.Z_{1}<-200 \mathrm{~cm}, 2\right)-200 \mathrm{~cm}<Z_{2}<0 \mathrm{~cm}, 0 \mathrm{~cm}<Z_{3}<200 \mathrm{~cm}$ and $200 \mathrm{~cm}<Z_{4}$. As an example let's take $Z_{3}$. The two arrival times for BMD-A and BMD-C are: $T_{A}=\frac{2 m+Z_{3}}{n c}$ and $T_{C}=\frac{2 m-Z_{3}}{n c}$, respectively, The quantity $n(<1)$ represents the percentage of the velocity that the particles travel with respect to the speed of light. $Z_{3}$ are the points in this Zone. Then we have:

$$
T_{A}-T_{C}=\frac{2}{n c} Z_{3}
$$

It means that the differences is propotional to de distance. If we take the $Z_{2}$ we will have the negative of Eq. 1. We see that the difference, in middle Zone, is linear with respect to the position of the interaction point. This simple calculation can be made for the other zones and for the sum. This theorical prediction are agree with the results obtained by MC, as is shown in Figure 2. Note that each operation has a different shape. Then, it is possible to distinguish if the collision occurs inside or outside BMD. In Figure2 are shown all arrival particles (primary and secondary).

\subsection{Arrival time resolution}

We worked inside BMD and selected the fastest particle for each event for BMD-A and BMD-C and we made a fit for their respective TA and TC. The fit was made with a Gaussian function, then, the difference in their $\sigma$ is the arrival time resolution, i.e.

$$
\Delta \sigma=\left|\sigma_{A}-\sigma_{C}\right|=57.982 \pm 0.509 p s
$$

The resolution time of all detector can be divided in three resolution times: 1) The arrival resolution times of particles from the collision (Eq. 2), 2) The arrival resolution time of the scintillator-photons created by the scintillator material of BMD and 3) The resolution time of the electronic. Reading this information must be done before the other event occurs. We expected that BMD will have a resolution time at most $50 \mathrm{ps}$.

\subsection{Geant4 simulation}

As is mentioned above, the circular geometry is not the final for the BMD, it was used as a preliminary results. The final geometry we will use is a hexagonal. We simulated Bc404 scintillator material in a hexagonal geometry (which represent a cell of BMD) using Geant4. In Figure 3 is shown the 


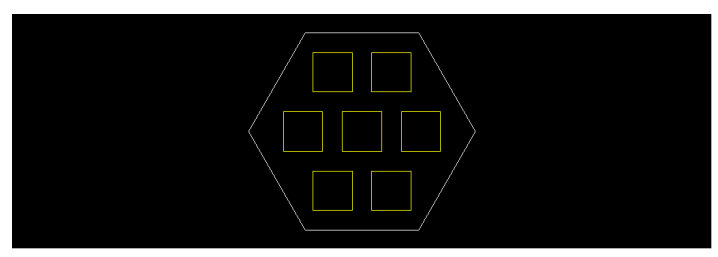

Figure 3. Ilustration for one scintillator cell of BMD-A and BMD-C. The yellow squares represent the APD to collect the scintillator photons. They are located behind the scintillator. Each one covers $2 \mathrm{~cm} \times 2 \mathrm{~cm}$ of area.

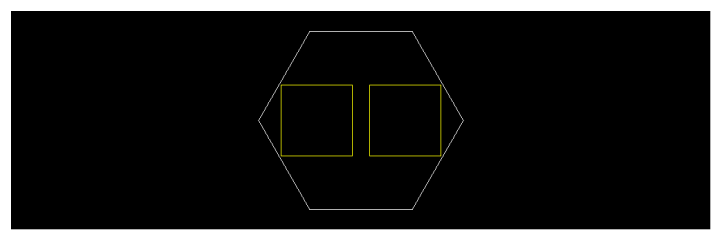

Figure 4. Same description as in Figure 3.

cell. The yellow squares are the representation of APDs. The dimentions of the scintillator material are $10 \mathrm{~cm} \times 10 \mathrm{~cm}$ and $2 \mathrm{~cm}$ of thick. The material we used is Bc404. We considered a $\pi^{+}$as a primary particle. The energy deposited is calculated by mpdroot and is arround $5 \mathrm{MeV}$, this results was obtained using the MPDroot simulation. We condired the fastest photon collectet by each APD and we obtained that $133.579 \pm 21.803 \mathrm{ps} \leq \Delta \sigma \leq 226.409 \pm 37.821 \mathrm{ps}$. This resolution time is too bigger for the ideal resolution time.

It is natural to think that if the cell is more smaller, the time resolution will be lower. We made the simulation considering the dimentions $5 \mathrm{~cm} \times 5 \mathrm{~cm}$ and $2 \mathrm{~cm}$. Due to the dimentions, it is only possible to use two scorers, as is shown in Figure 4. The rsolution time for this arrangement is $\Delta \sigma=$ $12.908 \pm 4.762 \mathrm{ps}$, which is obviously very good. However, we need to cover $1 \mathrm{~m} \times 1 \mathrm{~m}$ of area, so, using this dimentions, will be expensive. We will continue to this study, finding the geometry to obtain the ideal resolution time.

\section{Conclusions}

The implementation of BMD code in the mpdroot framework was successfully. With this new framework, we could calculate in first aproximation the time resolution for BMD. The circular geometry will not be the final geometry for BMD, however this results will not depend on it. The arrival resolution time analysis can be done for each cell to and choose the better sections or cells to detemine the better arrival resolution time and improve the resolution time of BMD. Also, with this "puntual" study can be used to determine the centrality, interaction point position, etc. And obtain a better measurement.

\section{References}

[1] L. Yordanova, J. Phys.: Conf. Ser. 503, 012041, (2014) 